\title{
A 10-year follow-up of the European multicenter trial of interferon beta-1b in secondary-progressive multiple sclerosis
}

Kuhle, J.

2016-04

Kuhle , J , Hardmeier, M , Disanto , G, Gugleta , K, Ecsedi , M , Lienert , C , Amato , M P , Baum , K, Buttmann , M , Bayas , A, Brassat , D , Brochet , B , Confavreux , C, Edan , G , Färkkilä, M , Fredrikson, S , Frontoni , M , D'Hooghe, M , Hutchinson, M , De Keyser , J , Kieseier , B C , Kuempfel , T, Rio , J , Polman , C , Roullet , E , Stolz , C , Vass , K, Wandinger , K P , Kappos , L \& European Long Term Follow Up Study 2016 , ' A 10-year follow-up of the European multicenter trial of interferon beta-1b in secondary-progressive multiple sclerosis ' , Multiple Sclerosis Journal , vol. 22 , no. 4 , pp. 533-543 . https://doi.org/10.1177/135245851559

http://hdl.handle.net/10138/161300

https://doi.org/10.1177/1352458515594440

publishedVersion

Downloaded from Helda, University of Helsinki institutional repository.

This is an electronic reprint of the original article.

This reprint may differ from the original in pagination and typographic detail.

Please cite the original version. 


\section{A 10-year follow-up of the European multicenter trial of interferon $\beta-1 b$ in secondary-progressive multiple sclerosis}

\author{
J Kuhle*, M Hardmeier*, G Disanto, K Gugleta, M Ecsedi, C Lienert, MP Amato, K Baum, \\ M Buttmann, A Bayas, D Brassat, B Brochet, C Confavreux, G Edan, M Färkkilä, S Fredrikson, \\ M Frontoni, M D'Hooghe, M Hutchinson, J De Keyser, BC Kieseier, T Kümpfel, J Rio, \\ C Polman, E Roullet, C Stolz, K Vass, KP Wandinger, L Kappos and the European Long-term \\ Follow-up Study Group in Interferon $\beta$-1b in Secondary-progressive Multiple Sclerosis \\ *J Kuhle and M Hardmeier contributed equally.
}

\section{Abstract}

Objectives: To explore long-term effects of treatment and prognostic relevance of variables assessed at baseline and during the European secondary progressive multiple sclerosis (SPMS) trial of interferon beta $1 \mathrm{~b}$ (IFNB-1b).

Methods: We assessed 362 patients (60\% female; median age 41 years; Expanded Disability Status Scale (EDSS): 5.5; 51\% randomized to IFNB-1b) for their EDSS and treatment history after 10 years. Nonparametric analysis of covariance (ANCOVA) and multivariate linear regression models were applied.

Results: Median EDSS was 6.0 at the end of the randomized controlled trial (RCT), in the IFNB-1b and placebo groups, and 7.0 in long-term follow-up patients (those receiving IFNB-1b in the RCT were 6.5 and those receiving placebo in the RCT were $7.0 ; p=0.086) .24$ patients $(6.6 \%)$ were deceased. The EDSS at baseline and the EDSS change during the RCT were the most important predictors of the EDSS 10 years later (partial $R^{2}: 0.47$ ). The ability to predict changes in EDSS 10 years after the RCT was limited $\left(R^{2}: 0.12\right)$. Magnetic resonance imaging (MRI) measures remained in the predictive models, but explained $<5 \%$ of the variability.

Conclusions: The results from this analysis did not provide convincing evidence to support a favorable long-term outcome in those patients allocated IFNB-1b during the RCT, in our SPMS cohort. The progressive stage of the disease remains largely unpredictable by clinical and conventional MRI measures, so better prognostic markers are needed.

Keywords: Disability, interferon beta, long-term outcome, magnetic resonance imaging, multiple sclerosis, neutralizing antibodies, prognosis, randomized controlled trial, secondary progressive multiple sclerosis

Date received: 6 January 2015; accepted: 20 May 2015

\section{Introduction}

Several aspects of secondary progressive multiple sclerosis (SPMS) are still poorly understood. Despite high inter-individual variability, natural history studies investigating large cohorts of MS patients reveal that SPMS begins after a median disease duration of about 19 years ${ }^{1,2}$ and that disease progression is continuous, after certain disability milestones are reached. ${ }^{3}$
Reliable prediction of onset and speed of progression could facilitate patient selection for treatment trials; however, the only reported predictors of slow disability accumulation in SPMS are the occurrence of relapses and a longer time to onset of progression..$^{2,4}$ Although distinctive imaging features are not well established, studies show a higher T2-lesion load in SPMS than in primary progressive MS (PPMS) ${ }^{5}$ and suggest distinctive T1 hypointense evolution and atrophy patterns in
Multiple Sclerosis Journal 2016, Vol. 22(4) 533-543

DOI: 10.1177 1352458515594440

(C) The Author(s), 2015. Reprints and permissions: http://www.sagepub.co.uk/ journalsPermissions.nav

Correspondence to: Ludwig Kappos

Neurology, Departments of Medicine, Clinical Research and Biomedicine, University Hospital Basel, Petersgraben 4, CH-4031 Basel,

Switzerland. ludwig.kappos@usb.ch

M Hardmeier

K Gugleta

M Ecsedi

L Kappos

Neurology, Departments of Medicine, Clinical Research and Biomedicine, University Hospital Basel

G Disanto

Blizard Institute, Queen Mary University of London, Barts, UK/London School of Medicine and Dentistry, UK. Neurocentre of Southern Switzerland, Ospedale

Civico, Lugano, Switzerland.

J Kuhle

Neurology, Departments of Medicine, Clinical Research and Biomedicine, University Hospital Basel/ Blizard Institute, Queen

Mary University of London, Barts, UK/London School of Medicine and Dentistry, UK University Hospital Basel,

Switzerland

C Lienert

Department of Medicine, Neurology, Kantonsspital Baselland Bruderholz, Switzerland.

MP Amato

Department NEUROFARBA, Section Neurosciences, University of Florence, Italy

K Baum

Department of Neurology, Klinik Hennigsdorf, Germany

M Buttmann

Department of Neurology, University of Würzburg, Germany

A Bayas Department of Neurology, Klinikum Augsburg, Germany D Brassat

Unité Institut national de la santé et de la recherche médicale (INSERM)

563, Centre hospitalier universitaire Purpan,

Toulouse, France 
B Brochet

Department of Neurology, and INSERM-CHU CIC-P 0005, Centre hospitalier universitaire de Bordeaux, France

C Confavreux

Centre de coordination EDMUS pour la sclérose en plaques, Hôpital Neurologique PierreWertheimer, Lyon, France

\section{G Edan}

Department of Neurology, Centre hospitalier universitaire Pontchaillou, Rennes, France

\section{Färkkilä}

Department of Neurology, Central Hospital, Helsinki

University, Finland

S Fredrikson

Karolinska University

Hospital Huddinge,

Karolinska Institutet,

Stockholm, Sweden

M Frontoni

Department of Neurological

Sciences, University of Rome

La Sapienza, Italy

M D'Hooghe

Department of Neurology,

National MS Center,

Melsbroek, Belgium/Vrije

Universiteit Brussel, Belgium

\section{Hutchinson}

Saint Vincent's University

Hospital, University College

Dublin, Ireland

J De Keyser

Departments of Neurology, University Medical Center Groningen, Netherlands

\section{BC Kieseier}

Department of Neurology,

Medical Faculty, Heinrich-

Heine University, Düsseldorf,

Germany

T Kümpfel

Institute of Clinical

Neuroimmunology, Ludwig

Maximilians University,

Munich, Germany

J Rio

MS Center of Catalonia

(Cemcat), Vall d'Hebron

University Hospital,

Barcelona, Spain

C Polman

MS Center Amsterdam, Vrije

University Medical Center,

Netherlands

E Roullet

Department of Neurology,

Hôpital Tenon, Paris, France

C Stolz

PAREXEL International

$\mathrm{GmbH}$, Berlin, Germany

K Vass

University Clinic of

Neurology, Medical

University of Vienna, Austria

KP Wandinger

University Hospital of

Schleswig-Holstein, Lübeck,

Germany
SPMS, in comparison to relapsing-remitting MS (RRMS) and PPMS. ${ }^{6}$ Furthermore, a small longitudinal study found that grey matter atrophy and T2-lesion load were the most relevant predictors for conversion to SPMS, in a 13-year follow-up. ${ }^{7}$

Treatment options in SPMS are limited. A positive effect of interferon-beta $1 \mathrm{~b}$ (IFNB-1b) was found in the European SPMS study (EU-SPMS), ${ }^{8}$ but this was not replicated in the North American SPMS trial, possibly due to differences in the selection of patients. ${ }^{9,10}$ It was not seen for IFNB-1a. ${ }^{11,12}$

Despite this, the long-term effects of these treatments in SPMS patients are not known. Long-term followup (LTFU) studies of randomized controlled trials (RCTs) in RRMS have often indicated beneficial outcomes in patients initially randomized to the active drug. ${ }^{13-16}$ Nevertheless, these studies are frequently criticized for methodological constraints, such as selection bias, i.e. the attrition of deteriorating patients, which potentially flaw the generalizability of their results ${ }^{17,18}$; however, because patients recruited for treatment trials are well characterized and prospectively followed during the RCT, clinically and by magnetic resonance imaging (MRI), they constitute a valuable source to elucidate prognostic factors and may still allow for limited estimations of long-term treatment effects. In a 10-year LTFU, we explored these two objectives in 362 patients originally included into the EU-SPMS trial. ${ }^{8}$

\section{Methods}

\section{EU-SPMS trial and open-label extension}

The EU-SPMS trial was a multicenter, double-blind trial comparing IFNB-1b to placebo, conducted on 718 patients between 1995 and $1998 .^{8}$ The patients were randomly assigned to receive either IFNB-1b (8 MIU) every other day $(n=360)$, or placebo $(n=358)$. After completion of the 3-year RCT, all patients were offered the chance to receive IFNB-1 in an open-label (OL) extension that lasted for an additional 18 months. Thereafter, treatment decisions were not protocoldefined, but at the discretion of the treating physicians and patients.

\section{Long-term follow-up: Patients and data collection} At the end of OL extension, all 35 EU-SPMS centers were asked to provide long-term observational data on their patients. After obtaining ethical approval and written informed consent, 23 centers took part in the LTFU and provided data by a standardized documentation

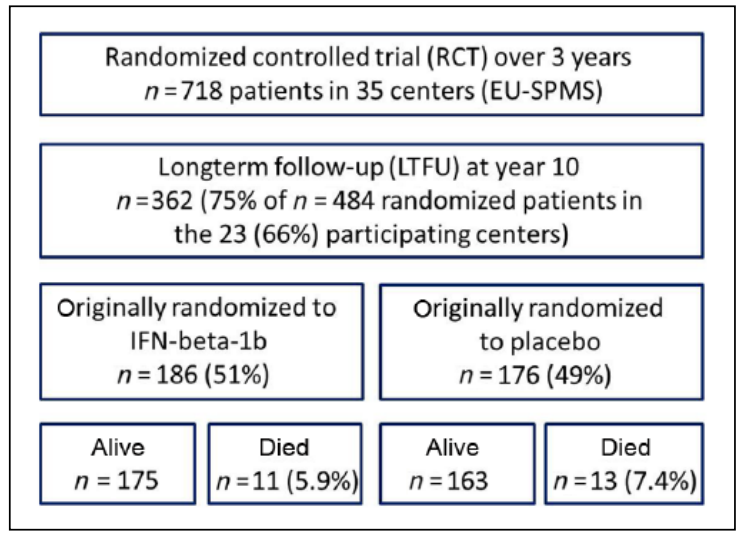

Figure 1. Patient disposition. The number of patients with a LTFU as part of the EU-SPMS cohort, their original treatment allocation and the number of deaths within the subgroups.

EU-SPMS: European SPMS study; LTFU: long-term follow-up; MS: multiple sclerosis; RCT: randomized controlled trial; SPMS: secondary progressive MS.

form, every 6 or 12 months. Demographic data, the patients' Expanded Disability Status Scale (EDSS) score, clinical status (i.e. stable or in relapse), and both the change in treatment and occurrence of relapses were captured. The EDSS values obtained during a relapse were excluded. EDSS values from 362 patients were documented between months 112 and 152 (a time-span referred to as Year 10). Of these 362 patients, 186 $(51.4 \%)$ were originally randomized to IFNB-1b and $176(48.6 \%)$ to placebo; while $24(6.6 \%)$ were deceased (Figure 1).

The baseline characteristics of the LTFU and nonLTFU patients are provided in Table 1. LTFU participants had a median EDSS of 5.5 (interquartile range (IQR): 4.0-6.0) divided into the following EDSS categories: EDSS 3.0-3.5 $(n=66)$, EDSS 4.0-5.5 $(n=$ $139)$ and EDSS 6.0-6.5 $(n=157)$.

We collected and analyzed the MRI measures centrally, according to standardized protocols. ${ }^{19}$ Notably, the EDSS is not a linear measure and the time during which scores increase is not equally distributed across the scale. ${ }^{20}$ Therefore, as in the EU-SPMS trial, the EDSS changes from EDSS 6.0 to 6.5, and from EDSS 6.5 to 7.0, were considered as equivalent to a 1.0-point change (see the supplementary Table S1).

\section{Statistics}

Continuous variables are presented as median with interquartile range (IQR); and categorical variables as 
Table 1. Comparison of baseline characteristics of (a) participants and non-participants within the LTFU centers, (b) all patients from participating LTFU centers, (c) non-participating centers (non-LTFU centers) and (d) all patients from all centers (EU-SPMS centers).

\begin{tabular}{|c|c|c|c|c|c|}
\hline & \multicolumn{2}{|c|}{ (a) LTFU centers $(n=484)$} & \multirow{2}{*}{$\begin{array}{l}\text { (b) LTFU centers } \\
(n=23)(65.7 \%) \\
n=484(67.4 \%)\end{array}$} & \multirow{2}{*}{$\begin{array}{l}\text { (c) Non-LTFU } \\
\text { centers }(n=12) \\
(34.3 \%) \\
n=234(32.6 \%)\end{array}$} & \multirow{2}{*}{$\begin{array}{l}\text { (d) EU-SPMS } \\
\text { centers }(n=35) \\
(100 \%) \\
n=718(100 \%)\end{array}$} \\
\hline & $\begin{array}{l}\text { participants } \\
n=362(74.8 \%)\end{array}$ & $\begin{array}{l}\text { non-participants } \\
n=122(25.2 \%)\end{array}$ & & & \\
\hline Gender female (\%) & 59.7 & 52.5 & 57.9 & $67.9(p=0.0113)^{\mathrm{b}}$ & 61.1 \\
\hline Age $(y)$ & 41.0 & 41.5 & 41.0 & 41.0 & 41.0 \\
\hline $\begin{array}{l}\text { Disease duration (y since first } \\
\text { symptoms) }\end{array}$ & 12.4 & 11.5 & 12.1 & 11.4 & 11.9 \\
\hline Age at onset of MS (y) & 27.0 & 28.0 & 27.0 & 28.0 & 27.0 \\
\hline Time since diagnosis of RRMS (y) & 6.7 & $5.4(p=0.0338)^{\mathrm{b}}$ & 6.4 & 6.6 & 6.4 \\
\hline $\begin{array}{l}\text { Patients without relapses in } \\
\text { previous } 2 \text { y }(\%)\end{array}$ & 32.0 & 29.5 & 31.4 & 27.4 & 30.1 \\
\hline Duration of progressive disease $(\mathrm{y})$ & 3.0 & 3.3 & 3.1 & 2.9 & 3.0 \\
\hline EDSS & 5.5 & 5.5 & 5.5 & $5.5(p=0.0088)^{\mathrm{b}}$ & 5.5 \\
\hline EDSS change in previous $2 y^{a}$ & 1.5 & $1.0(p=0.038)^{\mathrm{b}}$ & 1.5 & 1.0 & 1.5 \\
\hline $\mathrm{T} 2$ lesion volume $\left(\mathrm{cm}^{3}\right)$ & 20.6 & 24.6 & 21.4 & 24.5 & 22.1 \\
\hline \multicolumn{6}{|c|}{$\begin{array}{l}\text { aOnly used as predictor variable in a univariate regression model, as only assessed in } \mathrm{n}=275 \text { of the participating patients. } \\
\text { bP values }<0.1 \text { are displayed (Wilcoxon-rank-sum test for continuous, Fisher's exact test for categorical variables, for comparison between (a) participants and } \\
\text { non-participants within the LTFU centers, (b) between LTFU centers and (c) Non-LTFU centers). Values are medians or proportions. } \\
\text { EDSS: Expanded Disability Status Scale; EU-SPMS: the European SPMS study; LTFU: long-term follow-up; MS: multiple sclerosis; RRMS: relapsing-remit- } \\
\text { ting MS; y: years. }\end{array}$} \\
\hline
\end{tabular}

numbers and proportions. In order to explore bias in patient ascertainment, the baseline and on-trial characteristics were compared between the LTFU versus the non-LTFU centers, and participating versus nonparticipating patients (both overall and within the LTFU centers), by Fisher's exact test or Wilcoxon rank sum test.

In an intention-to-treat analysis, we compared the EDSS at Year 3 and Year 10 and the change in EDSS from baseline to Year 3 and Year 10, between the IFNB-1b and placebo groups, using the Wilcoxon test at baseline and a nonparametric analysis of covariance (ANCOVA) with covariate adjustment for baseline EDSS, at Year 3 and Year 10, as in the EU-SPMS trial.

We determined the prognostic factors for long-term outcome using multivariate linear regression models, in which the baseline (Table 1), RCT measures (Table 2) and the treatment allocation during the EU-SPMS were used as predictors of EDSS at Year 10 and the change in EDSS from the end of the RCT to Year 10. We employed a stepwise elimination procedure, with $p<0.5$ to enter and $p<0.05$ to stay in the model, to determine the weights of the most informative set of predictors in the final model. Multivariate models included $94.2 \%$ of the LTFU patients, due to the fact that in 21 patients the change in $\mathrm{T} 2$ lesion volume (T2 burden of disease (BOD)) was not available. The EDSS change in the 2 years prior to study inclusion was tested only in univariate models, as it was only assessed in a subgroup of patients $(n=275)$.

Deaths were considered as EDSS 10. The KaplanMeier curves of IFNB-1b and placebo patients were compared by a log-rank test. $P$-values lower than 0.05 were considered as statistically significant. We performed all statistical analyses using SAS version 9.2 and IBM SPSS, version 21.

\section{Results}

\section{Recruitment, treatment allocation and mortality}

The 23 LTFU centers had recruited 484 of 718 participants of the RCT (67\%) and provided follow-up data on 362 patients $(50 \%$ of all patients and $75 \%$ of patients within the LTFU centers). Patient ascertainment within the LTFU centers ranged from $30 \%$ to 100\%: We had 17 centers provide LTFU for $>70 \%$ and four centers provide LTFU for $100 \%$ of their randomized patients (Figure 1).

The proportion of treated patients and the type of treatment until Year 10 in the LTFU cohort, based on 
Table 2. Randomized controlled trial characteristics of (a) participants and non-participants within LTFU centers, (b) all patients from participating LTFU centers, (c) non-participating centers (non-LTFU centers) and (d) all patients from all centers (EU-SPMS centers).

\begin{tabular}{|c|c|c|c|c|c|}
\hline & \multicolumn{2}{|c|}{ (a) LTFU centers $(n=23)$} & \multirow{2}{*}{$\begin{array}{l}\text { (b) LTFU centers } \\
(n=23) \\
n=484\end{array}$} & \multirow{2}{*}{$\begin{array}{l}\text { (c) Non-LTFU } \\
\text { centers }(n=12) \\
n=234\end{array}$} & \multirow{2}{*}{$\begin{array}{l}\text { (d) EU-SPMS } \\
\text { centers }(n=35) \\
n=718\end{array}$} \\
\hline & $\begin{array}{l}\text { participants } \\
n=362\end{array}$ & $\begin{array}{l}\text { non-participants } \\
n=122\end{array}$ & & & \\
\hline Change in EDSS & 0.5 & 1.0 & 0.5 & 1.0 & 0.5 \\
\hline Confirmed progression $(\%)$ & 50.8 & 50.8 & 50.8 & 50.4 & 50.7 \\
\hline Patients with relapses $(\%)$ & 60.5 & 53.3 & 58.7 & $67.1(p=0.0334)^{\mathrm{a}}$ & 61.4 \\
\hline Patients becoming wheelchair-bound (\%) & 22.7 & 23.0 & 22.7 & 25.6 & 23.7 \\
\hline $\mathrm{RR}$ & 0.31 & 0.30 & 0.31 & $0.35(p=0.0017)^{\mathrm{a}}$ & 0.31 \\
\hline Change in T2 lesion volume, median (\%) & 1.79 & -1.63 & 0.22 & 1.58 & 0.96 \\
\hline Patients with $>1$ enlarging MRI lesions (\%) & 58.8 & 61.5 & 59.4 & 60.4 & 59.8 \\
\hline \multicolumn{6}{|c|}{$\begin{array}{l}\text { a } P \text { values }<0.1 \text { are displayed (Wilcoxon-rank-sum test for continuous, Fisher's exact test for categorical variables for comparison (a) between participants and } \\
\text { non-participants within LTFU centers; and between LTFU (b) centers and the Non-LTFU centers (c). Values are medians or proportions. } \\
\text { EDSS: Expanded Disability Status Scale; EU-SPMS: European SPMS study; LTFU: long-term follow-up; MRI: magnetic resonance imaging; MS: multiple } \\
\text { sclerosis; RR: relapse rate; RRMS: relapsing-remitting MS; y: years. }\end{array}$} \\
\hline
\end{tabular}

original randomization to IFNB-1b or placebo, is shown in Figure 2. At the end of the OL extension, there were $247(68 \%)$ patients on IFNB-1b and 120 patients $(33 \%)$ still remained on IFNB-1b at Year 10 . The number of patients without immunomodulatory treatment increased from $82(23 \%)$ at Month 54, to 160 $(44 \%)$ at Year 10 . The overall median for treatment exposure, i.e. the proportion between time on any immunomodulatory treatment and the time of observation, was $65.2 \%$. As expected, this was significantly different between the two original treatment groups (IFNB-1b: 79.3\%; placebo: $52.5 \%$; $p<0.0001$ ).

A total of 24 patients $(6.6 \%)$ were deceased at a median age of 50.2 years (40.6-55.0). Nine patients died within the first 5 years of the observation period; 11 patients had been randomized to IFNB-1b and 13 to placebo. Their causes of death were considered as MS-related in six and seven patients respectively, and non-MS-related in two and two patients, respectively, and were considered unknown in three and four patients whom were randomized to IFNB-1b and placebo, respectively. Occurrence of deaths was not significantly different between the original treatment groups (log-rank: $p=0.57)$.

\section{Sample characteristics of participating and non-participating patients}

Within the LTFU centers, participating patients had a longer time since having the diagnosis of RRMS, compared to non-participating patients (6.7 versus 5.4 years; $p=0.034$ ) and a higher EDSS change in the 2 years prior to study inclusion (1.5 versus 1.0 points, $p$
$=0.038)$. The latter was only assessed in a subgroup of the EU-SPMS cohort $(n=433)$, and was the only variable that was significantly different between the LTFU and all non-participating patients (1.5 versus 1.0 points, $p=0.016$ ).

The comparisons between all patients in the LTFU centers $(n=484)$ and non-LTFU centers $(n=234)$ showed only minor differences at baseline (Table 1): There was a slightly higher proportion of patients with relapses $(58.7 \%$ versus $67.1 \% ; p=0.0334)$ and a higher relapse rate $(0.31$ versus $0.35 ; p=0.0017)$ in the nonLTFU centers during the RCT (Table 2). Neutralizing antibodies (NAB) against IFNB-1b were detected in $31 \%$ of the LTFU patients with active treatment.

\section{Changes in EDSS from baseline to Year 10}

The median baseline EDSS score in the LTFU patients was 5.5 (4.0-6.0) and increased to 6.0 (4.5-6.5) at Year 3 (observation time: 36.2 months (33.5-38.3)); and was $7.0(6.0-8.0)$ at Year 10 (observation period: 124.0 months (120-128)). At Year 10, 31.2\% of patients were wheelchair-bound (EDSS 7.0-8.0), $14.1 \%$ were bed-ridden (EDSS 8.5-9.5) and 6.6\% had deceased (Figure 3 and Supplementary Figure S1). The median EDSS change was $0.5(0-1.5)$ points at Year 3 and $2.5(1.0-3.5)$ points at Year 10. EDSS decreased by 1 point or more in $5.2 \%$ of patients; was stable (decreased by 0.5 , no change or increased by 0.5 points) in $11.9 \%$ of patients; and increased by $1.0-1.5$ points in $17.4 \%$ of patients, by $2.0-2.5$ points in $27.1 \%$ of patients, and by $\geqslant 3.0$ points in $38.4 \%$ of patients. 


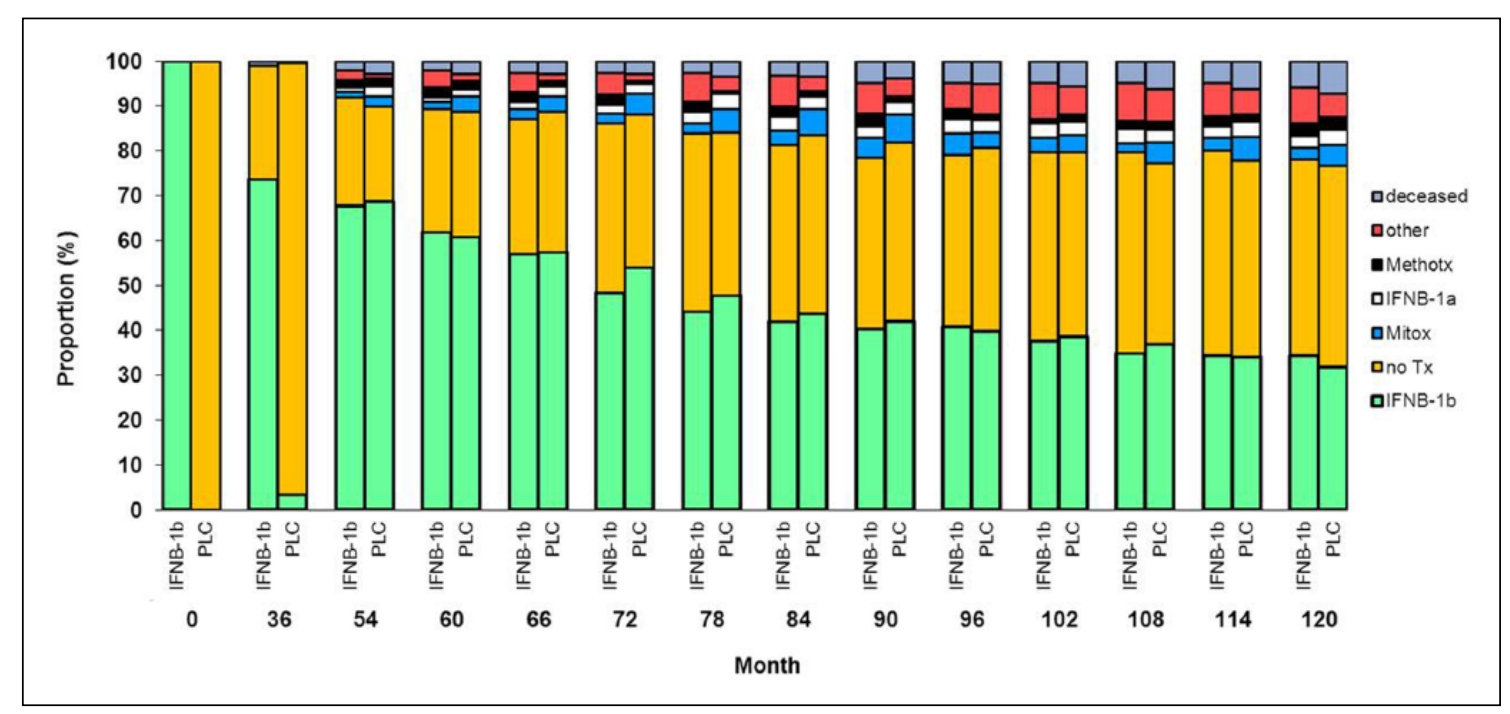

Figure 2. Usage of immunomodulatory treatments by patients participating in the 10-year follow-up, based on the original randomization to IFNB-1b or placebo in the RCT. At baseline (Month 0), 186 patients $(51.4 \%)$ had been randomized to IFNB-1b and 176 patients (48.6\%) to placebo. At Year 3 (Month 36), we had 143 (40\%) patients whom remained on IFNB-1b and $214(60 \%)$ patients whom had no treatment. At Month 54, at the end of the open-label extension, there were 247 patients $(68 \%)$ on IFNB-1b, 82 patients $(23 \%)$ on no treatment and 24 patients $(7 \%)$ on other treatments. At Year 10 (Month 120$)$, there were 120 patients (33\%) on IFNB-1b; 160 patients $(44 \%)$ had no treatment; and 13 patients $(4 \%)$ on mitoxantrone, 11 patients $(3 \%)$ on IFNB-1a IM, 10 patients $(3 \%)$ on methotrexate; while 24 patients $(7 \%)$ had switched to other alternative treatments (proportions include the deceased patients).

IFNB: interferon beta; IM: intramuscular; methotx: methotrexate; mitox: mitoxantrone; PLC: placebo; RCT: randomized controlled trial; Tx: treatment.

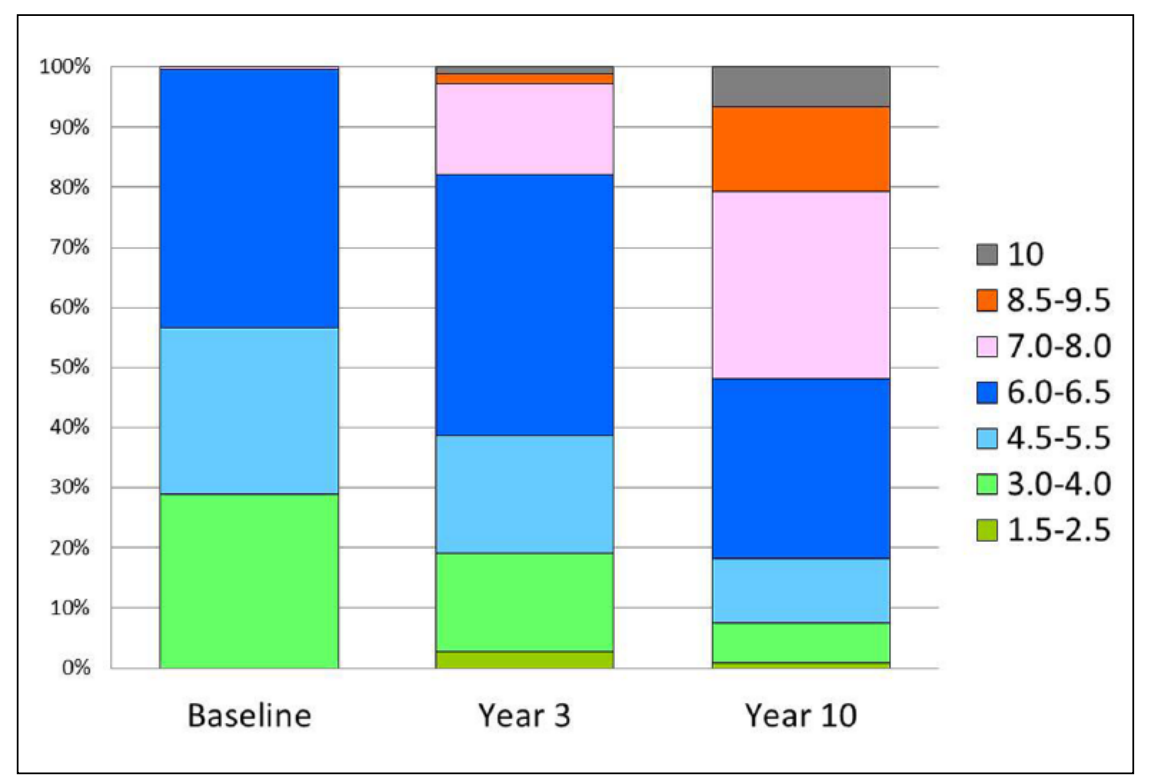

Figure 3. Proportion of EDSS categories over time in LTFU participants. Bars represent the proportion of patients of the LTFU sample in different EDSS grades $(n=362)$ at baseline, at the end of the RCT (Year 3) and at end of the LTFU (Year 10).

EDSS: Expanded Disability Status Scale; LTFU: long-term follow-up; RCT: randomized controlled trial.

There were no significant differences in EDSS between different treatment allocations at baseline (IFNB-1b: $5.5 \quad(4.0-6.0)$ versus placebo: 5.5
$(4.0-6.0) ; p=0.127)$ and at Year 3 (IFNB-1b: 6.0 (4.5-6.5) versus Placebo: $6.0(5.0-6.5) ; p=0.161$ (Figure 4)). There appeared to be a trend at Year 10, 


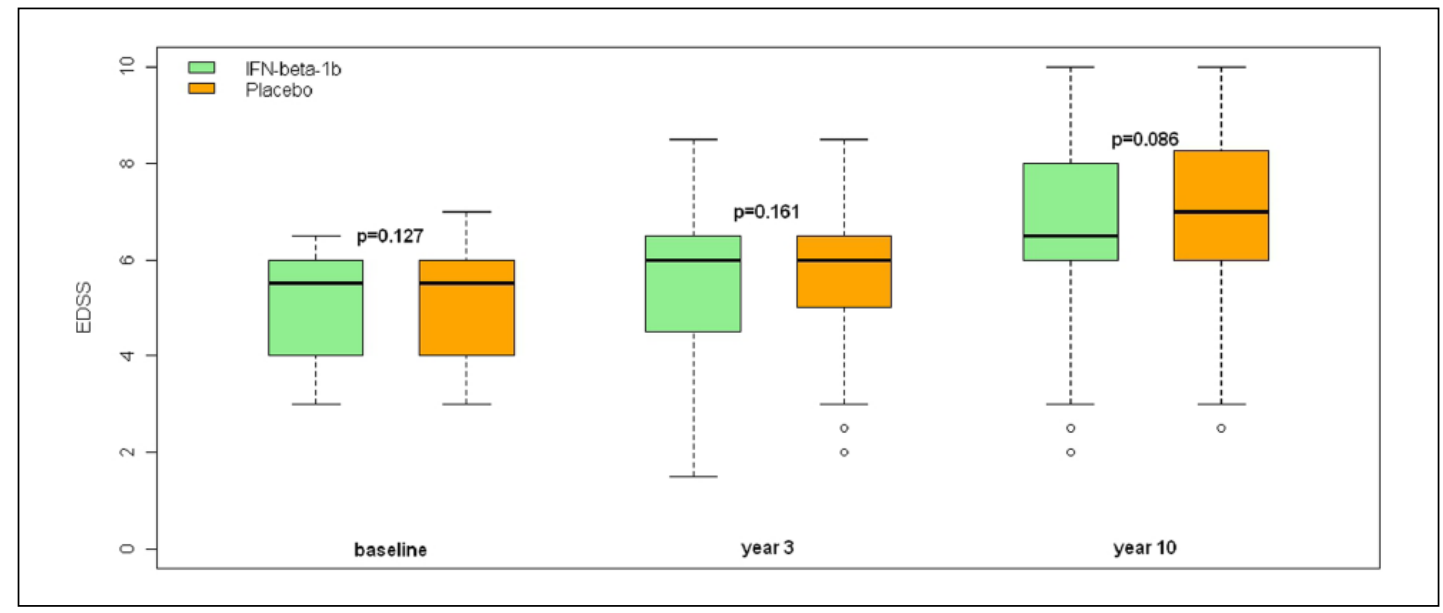

Figure 4. EDSS at baseline, at the end of the RCT (Year 3) and at end of LTFU (Year 10); by original treatment allocation $(n=362)$. Box plots represent EDSS of the LTFU sample $(n=362)$ by treatment group (IFNB-1b: $n=186$; Placebo: $n=176)$ at baseline, at Year 3 and Year 10 .

EDSS: Expanded Disability Status Scale; IFNB: interferon beta; LTFU: long-term follow-up period; RCT: randomized controlled trial.

in favor of the patients originally randomized to IFNB-1b (IFNB-1b: 6.5 (6.0-8.0) versus Placebo: $7.0(6.0-8.25) ; p=0.086)$; however, this trend was no longer visible when the deceased patients were excluded from the analysis $(p=0.13)$. The EDSS change at Year 3 and at Year 10 was not significantly different between the treatment groups (IFNB-1b: $0.5(0.0-1.5)$ and $2.5(1.0-3.5)$ points; Placebo: 1.0 $(0.0-2.0)$ and $2.5(1.0-3.5)$ points; $p=0.25$ and $p=$ 0.17 ). At Year 10, the proportion of patients reaching EDSS $\geqslant 8$ or death was not significantly different, between the two groups (IFNB-1B: 29\% versus Placebo: $36.4 \% ; p=0.29$ ).

\section{Prognostic factors for EDSS at year 10 and change in EDSS from end of RCT to year 10}

Prognostic factors for the patients' long-term outcome were determined using multivariate linear regression models. When considering only baseline variables, EDSS at Year 10 and the change in EDSS from the end of the RCT to Year 10 were both predicted by gender, T2 BOD and EDSS at baseline (Table 3). The overall proportion of variability in EDSS at Year 10, and the change in EDSS from the end of the RCT to Year 10, as explained by these models was $27.0 \%$ and $4.5 \%$, respectively. Baseline EDSS and T2 BOD explained the greatest proportion of variability in EDSS at Year $10(24.8 \%)$ and the change in EDSS from the end of the RCT to Year 10 (2.7\%), respectively (Table 3 ).

When the RCT outcome measures were included in the models, both long-term outcomes were predicted by the same variables: gender, baseline $\mathrm{T} 2$ BOD, change in T2 BOD during the RCT, baseline EDSS, and the change in EDSS during the RCT. It is noteworthy that treatment allocation was not retained in these models. Altogether, these variables explained $51.4 \%$ and $11.6 \%$ of the total variability in EDSS at Year 10 and the change in EDSS from the end of the RCT to Year 10, respectively. The change in EDSS during the RCT was the main predictor in both models (EDSS at Year 10: 24.2\%; change in EDSS to Year 10: 3.2\%) (Table 3). Notably, EDSS at baseline and the change in EDSS during the RCT had a negative regression coefficient in the models predicting change in EDSS from the end of the RCT to Year 10. In all models, having the female gender had a small protective effect. The EDSS change in the 2 years prior to study inclusion (assessment only in a subgroup, $n=275$ ) was not a significant predictor of long-term outcomes in our univariate analysis.

Finally, we tested the effect of baseline and RCT outcome measures on patient disability outcomes, only within the subgroup of IFNB-1b-treated patients in the RCT (Table 3). In contrast to the models run on the whole LTFU cohort, the relapse rate during the $\mathrm{RCT}$ and presence of more than one enlarging lesion on MRI during the RCT were weak predictors of EDSS at Year 10 and the change in EDSS from the end of the RCT to Year 10. The overall proportion of variability in EDSS at Year 10 and the change in EDSS from the end of the RCT to Year 10, as explained by these models, were $51.8 \%$ and $11.7 \%$, respectively (Table 3 ). 
Table 3. Final model of EDSS at Year 10 and the changes in EDSS from the end of the RCT to Year 10, in all LFTU patients and those randomized to IFNB-1b.

\begin{tabular}{|c|c|c|c|c|c|c|c|}
\hline \multirow[t]{2}{*}{ Model } & \multirow[t]{2}{*}{ Variables } & \multicolumn{3}{|c|}{ Predicting EDSS at Year 10} & \multicolumn{3}{|c|}{$\begin{array}{l}\text { Predicting a change in EDSS from the } \\
\text { end of the RCT to Year } 10\end{array}$} \\
\hline & & Coefficient & $P$ & $R^{2}(\%)$ & Coefficient & $P$ & $R^{2}(\%)$ \\
\hline \multirow{5}{*}{$\begin{array}{l}\text { Baseline measures for all } \\
\text { LFTU patients }\end{array}$} & Gender (female) & -0.335 & 0.033 & 1.0 & -0.37 & 0.0211 & 1.7 \\
\hline & IFNB- $1 \mathrm{~b}$ treatment & -0.378 & 0.015 & 1.1 & - & - & - \\
\hline & $\mathrm{T} 2 \mathrm{BOD}$ & 0.013 & 0.0008 & 2.5 & 0.010 & 0.0141 & 1.2 \\
\hline & EDSS & 0.655 & $<0.0001$ & 22.3 & -0.17 & 0.0209 & 1.5 \\
\hline & & & & 27.0 & & & 4.5 \\
\hline \multirow{6}{*}{$\begin{array}{l}\text { Baseline }+ \text { on-RCT } \\
\text { measures for all LFTU } \\
\text { patients }\end{array}$} & Gender (female) & -0.304 & 0.018 & 0.8 & -0.366 & 0.019 & 1.5 \\
\hline & T2 BOD & 0.01 & 0.002 & 1.5 & 0.012 & 0.0017 & 1.8 \\
\hline & T2 BOD change & 0.01 & $<0.0001$ & 2.2 & 0.011 & 0.0004 & 2.9 \\
\hline & EDSS & 0.679 & $<0.0001$ & 22.7 & -0.191 & 0.0075 & 2.2 \\
\hline & EDSS change & 0.569 & $<0.0001$ & 24.2 & -0.28 & $<0.0001$ & 3.2 \\
\hline & & & & 51.4 & & & 11.6 \\
\hline \multirow{7}{*}{$\begin{array}{l}\text { Baseline }+ \text { on-RCT } \\
\text { measures (IFNB-1b treated } \\
\text { patients) }\end{array}$} & $>1$ enlarging lesion & 0.392 & 0.042 & 1.2 & 0.602 & 0.0104 & 2.6 \\
\hline & $\mathrm{RR}$ & -0.387 & 0.017 & 1.3 & -0.530 & 0.0072 & 2.6 \\
\hline & T2 BOD change & 0.013 & 0.011 & 2.3 & - & - & - \\
\hline & T2 BOD & 0.014 & 0.003 & 2.5 & 0.016 & 0.0066 & 2.7 \\
\hline & EDSS & 0.618 & $<0.0001$ & 20.0 & -0.282 & 0.0067 & 3.9 \\
\hline & EDSS change & 0.601 & $<0.0001$ & 24.7 & - & - & - \\
\hline & & & & 51.8 & & & 11.7 \\
\hline
\end{tabular}

The influence of MRI measures on both outcomes appeared slightly stronger in the IFNB-1b subgroup than in the entire sample, but still explained only $6.0 \%$ and $5.3 \%$ of the variability of EDSS seen at Year 10 and the change in EDSS from the end of the RCT to Year 10, respectively. Notably, a higher relapse rate was inversely associated with both disability outcomes in IFNB-1btreated patients, as was baseline EDSS with the EDSS change from the end of the RCT to Year 10. The presence of NAB had no influence on either outcome.

\section{Discussion}

This study reports the results of the 10-year multicenter long-term follow-up of 362 patients with SPMS whom participated in the EU-SPMS treatment trial that tested IFNB-1b; it can be considered as a 'treated history' study. ${ }^{8}$ We performed an intention-to-treat-analysis to explore whether treatment allocation during the RCT had a long-term advantage; and we investigated which baseline and within-RCT variables were associated with disability scores, and the change in disability scores, after the 10-year follow-up.

In contrast to the whole sample, the LTFU-cohort showed no significant group differences in EDSS between the IFNB-1b and Placebo groups of patients, at Year $3 .^{8}$ This may be due to reduced statistical power and the influence of unknown variables, since the baseline and on-trial characteristics showed no relevant differences, in comparison with the nonparticipating patients. A trend in favor of initial IFNB-1b treatment was observed at Year 10, but this was no longer visible when the 24 deceased patients were removed from the analysis.

Long-term observational studies are prone to selection bias, as patients with more progressive disease are more likely to drop from the study. ${ }^{17,18}$ In addition, treatment effects in observational study designs are difficult to interpret: Unblinding, EDSS assessments without later confirmation and treatment decisions taken at the discretion of the physician represent potential sources of bias. In our study, selection bias may have been limited, because $75 \%$ of the patients within the participating centers were followed and the initial randomization was balanced between centers.

We found that 24 of $362(6.6 \%)$ patients died during the 10-year observation period, without differences by initial treatment allocation. Given an excess death rate of 13 per 1000 person-years in $\mathrm{MS},{ }^{21}$ more deaths 
could have been expected; however, as death rates have been observed to rise only 20 years after disease onset, ${ }^{22}$ the observation period of this study (about 22 years after disease onset) may be still too short to draw conclusions about mortality outcomes.

We found that the most relevant predictors of EDSS at Year 10 were baseline EDSS and EDSS changes during the RCT. Similarly, changes in EDSS between the end of the RCT and Year 10 were mainly predicted by changes in the EDSS during the first 3 years, albeit explaining only $3.2 \%$ of variability. Interestingly, a high EDSS score at baseline and a change in EDSS during the RCT were negatively associated with EDSS changes at the end of the follow-up period. This finding is likely explained by the non-linearity of the EDSS scale and the fact that the time needed to increase in EDSS category tends to be longer, in patients with higher EDSS scores..$^{20}$ In other words, the probability to progress in EDSS is higher at lower disability scores, and this is not entirely accounted for by considering the changes from EDSS 6.0 to 6.5 and from 6.5 to 7.0 as equivalent to a 1.0-point change, as we did.

Treatment allocation was a marginal predictor only in the model including baseline variables and predicting EDSS at Year 10, but not the change in EDSS from the end of the RCT to Year 10, corroborating the lack of convincing evidence for a more favorable longterm outcome in patients initially treated with IFNB-1b. Treatment exposure during the OL extension and the LTFU was not included in the model, as this variable is probably biased by selection: Stopping treatment due to ineffectiveness is more likely in the progressing than in the stable patients.

Notably, the contribution of the MRI and pre-RCT disease activity measures were only marginal, if not absent. Considering the results reported in the RCT, the weak contribution of conventional MRI predictors is not surprising. ${ }^{19,23}$ Even in RRMS, where inflammation is more prominent, long-term outcomes are only weakly predicted by conventional MRI measures $^{13}$; however, the analyses in the current study are limited to $\mathrm{T} 2$ lesion metrics, as $\mathrm{T} 1$ gadoliniumenhancing and $\mathrm{T} 1$ hypointense lesions, as well as brain atrophy, were only assessed in small subgroups of the EU-SPMS patients. ${ }^{24,25}$

While the clinical and MRI markers of disease activity have been proposed as markers of treatment response to IFNB in RRMS, ${ }^{26,27}$ our results suggested that they may be less relevant in the secondary progressive phase of the disease. Indeed, prognostic markers in the
IFNB-1b subgroup were essentially the same as in the entire cohort, with only slightly more weight on MRI measures; and notably, even an apparent protective effect of a higher relapse rate under treatment. However, gadolinium-enhancing lesions were only assessed in a subgroup of the randomized population and were therefore not included in this analysis. In RR MS gadolinium enhancing lesions have been described to be the strongest predictors of long-term treatment response. ${ }^{26}$

We observed that NAB status had no significant effect on the long-term disability outcome. Similarly, the NAB status had no significant effect on disease progression during the randomized controlled phase of both the EU-SPMS and the North American SPMS trials. ${ }^{10,28}$

Natural history studies suggest that predictors of the time to reach the progressive phase are no longer predictive, once the progression has started..$^{29-31}$ During progression, a longer time to reach DSS 3 and the occurrence of relapses are associated with delayed disability milestones. ${ }^{2}$ In our LTFU cohort, relapses during the RCT were negatively associated with long-term disability, only in the IFNB-1b-treated subgroup, while the duration of progressive disease was not predictive at all. The persistence of relapses under and despite IFNB-1b therapy may indicate the presence of a still more inflammatory disease, in which neurodegeneration may be less prominent; however, this is a speculation and these observations remain difficult to explain.

In summary, the progressive phase of MS remains largely unpredictable by baseline initial on study conventional MRI measures, and the long-term outcome is mostly predicted by the baseline disability and its change in the short term. Advanced MRI markers of tissue destruction may better predict long-term disability, as was recently shown for grey matter atrophy, ${ }^{7}$ but not for global brain atrophy nor width of third ventricle at baseline, in RRMS. ${ }^{13,26}$ The inclusion of other biomarkers derived from cerebrospinal fluid and blood samples, ${ }^{32}$ retinal nerve fibre thickness, ${ }^{33}$ as well as neurophysiological markers, ${ }^{34,35}$ may improve characterization of progressive MS.

In conclusion, the current study showed that the SPMS phase remains largely unpredictable by existing conventional clinical and paraclinical measures.

\section{Acknowledgements}

We are grateful to Bayer Health Care employees Joerg P Bugge and Karola Beckmann, for providing scientific and statistical advice. The leading and corresponding authors had full access to all the 
study data and had final responsibility for the decision to submit the paper for publication. We are indebted to all MS patients whom participated in this study.

\section{Conflict of interest}

J Kuhle was supported by an ECTRIMS Research Fellowship Programme and by the "Forschungsfonds" of the University of Basel, Switzerland and has received research support from the Swiss MS Society, Genzyme and Novartis and served in scientific advisory boards for Genzyme/Sanofi-Aventis, Merck Serono and Novartis Pharma;

M Hardmeier reports no disclosures;

G Disanto reports no disclosures;

K Gugleta reports no disclosures;

M Ecsedi reports no disclosures;

C Lienert has received travel support from Teva, Merck-Serono, Biogen, Bayer Schering;

MP Amato has received research grants and honoraria as a speaker and member of advisory boards by Bayer, Biogen Idec, Merck Serono, Novartis, Sanofi Genzyme, Teva, Almirall;

$\mathrm{K}$ Baum has received honoraria for lectures and consulting from Almirall, Bayer, Biogen, Merck Serono, Novartis, Roche, Sanofi-Genzyme.

M Buttmann received travel grants, consulting and/or speaker's fees from Bayer, Biogen, Genzyme, Merck Serono, Novartis, Roche, Teva, he received research grants from Merck Serono and Novartis;

A Bayas received honoraria for consulting and/or as speaker from Merck Serono, Biogen, Bayer Vital, Novartis, Sanofi/Genzyme, Roche and TEVA, for trial activities from Merck Serono, Biogen and Novartis and received grants for congress trips and participation from Bayer Vital, Novartis, Biogen, Sanofi/Genzyme and Merck Serono;

D Brassat reports no disclosures;

B Brochet serves on scientific advisory boards for and has received honoraria or research support from Biogen Idec, Merck Serono, Sanofi-Genzyme, Novartis, Bayer Schering Pharma, Teva and Roche;

G Edan reports no disclosures;

M Färkkilä reports no disclosures;

S Fredrikson has received honoraria for lectures, educational activities or consultancy from Allergan, Bayer, BiogenIdec, Genzyme, Merck, Novartis, Sanofi, Teva;

M Frontoni reports no disclosures;
M D'Hooghe received research support from Teva and Bayer and honoraria as a speaker and for participating in scientific advisory boards for GenzymeSanofi, Merck-Serono, Novartis, Biogen-Idec, Bayer and Allergan;

M Hutchinson reports no disclosures;

J De Keyser reports no disclosures;

BC Kieseier reports no disclosures;

T Kümpfel reports no disclosures;

J Rio reports no disclosures;

C Polman reports no disclosures;

C Stolz is an employee of PAREXEL International $\mathrm{GmbH}$, Berlin, Germany;

KP Wandinger reports no disclosures;

L Kappos has participated in the last 24 months as member or chair of planning and steering committees or advisory boards as well as independent safety boards, in most cases in conjunction with corporatesponsored clinical trials in multiple sclerosis and other neurological diseases. The sponsoring pharmaceutical companies for these activities include Addex, Actelion, Almirall, Bayer Health Care Pharmaceuticals, Biogen Idec, CLC Behring, Genentech, GeNeuro SA, Genzyme, Merck Serono, Mitsubishi Pharma, Novartis, Octopharma, Ono Pharma, Pfizer, Receptos, Roche, sanofi-aventis, Santhera, Siemens, Teva, UCB and Xenoport. L Kappos is principal investigator for the following drug studies BOLD, BOLD EXT., EXPAND (BAF312, Novartis), DECIDE, DECIDE EXT. (Daclizumab, Biogen Idec), ENDORSE (BG00012, Biogen Idec), FINGORETT, FTY-UMBRELLA, INFORMS, INFORMS EXT LONGTERM. (Fingolimod, Novartis), OCRELIZUMAB PHASE II EXT., OPERA, ORATORIO (Ocrelizumab, Roche), STRATA EXT. (Natalizumab, Biogen Idec) and TERIFLUNOMIDE EXT. (Teriflunomide, SanofiAventis). L Kappos lectured at medical conferences or in public on various aspects of the diagnosis and management of multiple sclerosis. In many cases these talks have been sponsored by non-restricted educational grants to my institution from one or another of the above listed companies. Honoraria and other payments for all these activities have been exclusively used for funding of research of my department. Research and the clinical operations (nursing and patient care services) of the MS Center in Basel have been supported by non-restricted grants from one or more of these companies and by grants from the Swiss MS Society, the Swiss National Research Foundation, the European Union, the Gianni Rubatto, Novartis and Roche Research Foundations. 


\section{Funding}

This work was supported by Bayer Pharma AG. Author JK was supported by the Swiss MS Society; Swiss ALS Society; European Committee For Treatment And Research In Multiple Sclerosis ('Research Fellowship Programme'); and by the University of Basel, Switzerland ('Forschungsfonds'). Author $\mathrm{MH}$ was supported by the Swiss National Science Foundation (grant numbers 33CM30-124115 and 33CM30-140338) and the Swiss MS Society.

\section{Notes}

Authors Kuhle and Hardmeier contributed equally to this work.

Authors Confavreux, Roulet and Vass were deceased by the end of the preparation of this manuscript.

The members of the European Long-term Follow-up Study Group in Interferon $\beta-1 \mathrm{~b}$ in Secondaryprogressive MS are shown in the supplementary material.

\section{References}

1. Weinshenker BG, Bass B, Rice GP, et al. The natural history of multiple sclerosis: A geographically-based study. I. Clinical course and disability. Brain 1989; 112: 133-146.

2. Vukusic S and Confavreux C. Prognostic factors for progression of disability in the secondary progressive phase of multiple sclerosis. J Neurol Sci 2003; 206: 135-137.

3. Confavreux C, Vukusic S and Adeleine P. Early clinical predictors and progression of irreversible disability in multiple sclerosis: An amnesic process. Brain 2003; 126: 770-782.

4. Scalfari A, Neuhaus A, Degenhardt A, et al. The natural history of multiple sclerosis, a geographically based study 10: relapses and long-term disability. Brain 2010; 133: 1914-1929.

5. Thompson AJ, Kermode AG, Wicks D, et al. Major differences in the dynamics of primary and secondary progressive multiple sclerosis. Ann Neurol 1991; 29: $53-62$.

6. Nijeholt GJ, Van Walderveen MA, Castelijns JA, et al. Brain and spinal cord abnormalities in multiple sclerosis. Correlation between MRI parameters, clinical subtypes and symptoms. Brain 1998; 121: 687-697.

7. Filippi M, Preziosa P, Copetti M, et al. Gray matter damage predicts the accumulation of disability 13 years later in MS. Neurology 2013; 81: 1759-1767.

8. European Study Group on Interferon Beta- $1 \mathrm{~b}$ in Secondary Progressive MS. Placebo-controlled multicentre randomised trial of interferon beta- $1 \mathrm{~b}$ in treatment of secondary progressive multiple sclerosis. Lancet 1998; 352: 1491-1497.

9. Kappos L, Weinshenker B, Pozzilli C, et al. Interferon beta- $1 \mathrm{~b}$ in secondary progressive MS: A combined analysis of the two trials. Neurology 2004; 63: 1779-1787.

10. Panitch H, Miller A, Paty D, et al. Interferon beta-1b in secondary progressive MS: Results from a 3-year controlled study. Neurology 2004; 63: 1788-1795.

11. Randomized controlled trial of interferon-beta-1a in secondary progressive MS: Clinical results. Neurology 2001; 56: 1496-1504.

12. Andersen O, Elovaara I, Farkkila M, et al. Multicentre, randomised, double-blind, placebocontrolled, Phase III study of weekly, low-dose, subcutaneous interferon beta-1a in secondary progressive multiple sclerosis. J Neurol Neurosurg Psychiatry 2004; 75: 706-710.

13. Goodin DS, Traboulsee A, Knappertz V, et al Relationship between early clinical characteristics and long-term disability outcomes: 16-Year cohort study (follow-up) of the pivotal interferon beta- $1 \mathrm{~b}$ trial in multiple sclerosis. J Neurol Neurosurg Psychiatry 2012; 83: 282-287.

14. Kappos L, Traboulsee A, Constantinescu C, et al Long-term subcutaneous interferon beta-1a therapy in patients with relapsing-remitting MS. Neurology 2006; 67: 944-953.

15. Rudick RA, Cutter GR, Baier M, et al. Estimating long-term effects of disease-modifying drug therapy in multiple sclerosis patients. Mult Scler 2005; 11: 626-634.

16. Ford C, Goodman AD, Johnson K, et al. Continuous long-term immunomodulatory therapy in relapsing multiple sclerosis: Results from the 15-year analysis of the US prospective open-label study of glatiramer acetate. Mult Scler 2010; 16: 342-350.

17. Noseworthy JH. How much can we learn from longterm extension trials in multiple sclerosis? Neurology 2006; 67: 930-931.

18. Marriott JJ and O'Connor PW. Lessons learned from long-term multiple sclerosis treatment trials. Mult Scler 2010; 16: 1028-1030.

19. Molyneux PD, Barker GJ, Barkhof F, et al. ClinicalMRI correlations in a European trial of interferon beta- $1 \mathrm{~b}$ in secondary progressive MS. Neurology 2001; 57: 2191-2197.

20. Pittock SJ, Mayr WT, McClelland RL, et al. Disability profile of MS did not change over 10 years in a population-based prevalence cohort. Neurology 2004; 62: 601-606. 
21. Bronnum-Hansen H, Koch-Henriksen N and Stenager E. Trends in survival and cause of death in Danish patients with multiple sclerosis. Brain 2004; 127: 844-850.

22. Goodin DS, Reder AT, Ebers GC, et al. Survival in MS: A randomized cohort study 21 years after the start of the pivotal IFNbeta-1b trial. Neurology 2012; 78: 1315-1322.

23. Li DK, Held U, Petkau J, et al. MRI T2 lesion burden in multiple sclerosis: A plateauing relationship with clinical disability. Neurology 2006; 66: 1384-1389.

24. Molyneux PD, Kappos L, Polman C, et al. The effect of interferon beta-1b treatment on MRI measures of cerebral atrophy in secondary progressive multiple sclerosis. Brain 2000; 123: 2256-2263.

25. Barkhof F, Van Waesberghe JH, Filippi M, et al. T(1) hypointense lesions in secondary progressive multiple sclerosis: Effect of interferon beta-1b treatment. Brain 2001; 124: 1396-1402.

26. Bermel RA, You X, Foulds P, et al. Predictors of longterm outcome in multiple sclerosis patients treated with interferon beta. Ann Neurol 2013; 73: 95-103.

27. Rio J, Castillo J, Rovira A, et al. Measures in the first year of therapy predict the response to interferon beta in MS. Mult Scler 2009; 15: 848-853.

28. Polman C, Kappos L, White R, et al. Neutralizing antibodies during treatment of secondary progressive MS with interferon beta-1b. Neurology 2003; 60: 37-43.
29. Weinshenker BG, Rice GP, Noseworthy JH, et al. The natural history of multiple sclerosis: A geographically-based study. 3. Multivariate analysis of predictive factors and models of outcome. Brain 1991; 114: 1045-1056.

30. Confavreux C, Vukusic S and Adeleine P. Early clinical predictors and progression of irreversible disability in multiple sclerosis: An amnesic process. Brain 2003; 126: 770-782.

31. Pittock SJ, Mayr WT, McClelland RL, et al. Change in MS-related disability in a population-based cohort: A 10-year follow-up study. Neurology 2004; 62: 51-59.

32. Gaiottino J, Norgren N, Dobson R, et al. Increased neurofilament light chain blood levels in neurodegenerative neurological diseases. PLoS One 2013; 8: e75091.

33. Petzold A, De Boer JF, Schippling S, et al. Optical coherence tomography in multiple sclerosis: A systematic review and meta-analysis. Lancet Neurol 2010; 9: 921-932.

34. Fuhr P, Borggrefe-Chappuis A, Schindler C, et al. Visual and motor evoked potentials in the course of multiple sclerosis. Brain 2001; 124: 2162-2168.

35. Schlaeger R, Schindler C, Grize L, et al. Combined visual and motor evoked potentials predict multiple sclerosis disability after 20 years. Mult Scler 2014; 20: $1348-1354$
Visit SAGE journals online http://msj.sagepub.com

↔SAGEjournals 